\title{
La signification, la tâche et la technique muséographique des collections d'instruments de musique
}

Curt Sachs

\section{OpenEdition}

\section{Journals}

Édition électronique

URL : http://journals.openedition.org/ethnomusicologie/573

ISSN : 2235-7688

Éditeur

ADEM - Ateliers d'ethnomusicologie

Édition imprimée

Date de publication : 1 novembre 2003

Pagination : 11-41

ISBN : 978-2-8257-0863-7

ISSN : $1662-372 X$

\section{Référence électronique}

Curt Sachs, "La signification, la tâche et la technique muséographique des collections d'instruments de musique », Cahiers d'ethnomusicologie [En ligne], 16 | 2003, mis en ligne le 16 janvier 2012, consulté le 20 avril 2019. URL : http://journals.openedition.org/ethnomusicologie/573

Ce document a été généré automatiquement le 20 avril 2019

Tous droits réservés 


\title{
La signification, la tâche et la technique muséographique des collections d'instruments de musique
}

\author{
Curt Sachs
}

1 Ce texte a été rédigé par Curt Sachs ${ }^{1}$ en français alors qu'il était attaché comme collaborateur permanent au Musée d'Ethnographie du Trocadéro à Paris, le futur Musée de l'Homme. Il y séjourna en effet de novembre 1933 à juillet 1937, à l'invitation de Paul Rivet, «pour collaborer à la classification des instruments de musique [...] avec André Schaeffner ». Déchu de toutes ses positions par le régime nazi en septembre 1933, il signe cet article comme «Ancien Directeur du Musée instrumental de Berlin ». Cette prise de position s'appuie en effet sur sa longue expérience au sein des collections instrumentales largement savantes et européennes de la Staatlichen Musikhochschule dont il eut la responsabilité à partir de $1919^{2}$.

Si l'on exclut la partie concernant la conservation et la restauration des instruments de musique, qui apparaît aujourd'hui pour une grande part dépassée compte tenu du développement des techniques et de la déontologie ${ }^{3}$, on remarquera que cette réflexion prend d'emblée en compte l'ouïe et la vue, focalise le débat sur «l'idée dominante de l'installation » et non sur la collection, constituant ainsi un véritable manifeste dont on ne voit pas qu'il ait été remplacé: tandis que les réalisations ont été multiples ces dernières décennies, combien de leurs auteurs ont en effet proposé une théorie de leur action muséographique? Sachs écrivit ce texte alors que le Musée de l'Homme était en gestation. Beaucoup de ses propositions vont bien au delà de la seule conception « esthétique » de la musique occidentale évoquée dans les salles du musée de Berlin. Elles reflètent les préoccupations d'un ethnomusicologue « universaliste » portant un regard prospectif à un moment crucial de l'évolution de la muséographie. Cette prise de position eut-elle une influence sur les salles musicales ouvertes peu après par André Schaeffner au Musée de l'Homme? Alors que cette page d'histoire est en train de se tourner et que de nouvelles réalisations muséographiques sont programmées, les perspectives ouvertes par ce texte nous invitent à conceptualiser nos propres convictions et introduisent fort à 
propos les éléments du débat ici publiés.

Florence Gétreau

2 De la naïve collection d'objets " curieux », tel cet ensemble hétéroclite qu'avait rassemblé une héroïne de Gottfried Keller, dans les "Gens de Seldwyla », aux prétentieux cabinets de curiosité des princes de la Renaissance, il n'y a pas si loin. Dans les deux cas, un même principe s'affirme : la recherche des objets qui nous élèvent au-dessus de nous-mêmes, pour le plaisir de notre imagination et pour le besoin que nous avons de transformer le passé et le lointain en présent et en proche, au moyen de la possession des objets qui sont le témoignage d'une époque ou d'une région lointaine.

De semblables motifs, cependant, ne nous suffisent plus. Nous cherchons à donner un but à la collection quelle qu'elle soit, but qui la justifie et récompense les efforts et les frais qu'elle occasionne. Dès que l'on étend le problème à la collection publique, il faut se demander tout d'abord à quel genre de public on désire s'adresser; sera-ce à tous les visiteurs, profanes et initiés, ou aux seuls spécialistes, aux artistes, aux savants ? Puis, une fois cette question résolue, on se demandera quelle doit être la fonction du musée: tendra-t-elle à instruire ou seulement à récréer, à initier ou à distraire? En d'autres termes, fera-t-on appel à l'intelligence ou au sentiment, ou bien à tous les deux à la fois ? Conservera-t-on des reliques ou des documents? Autant de problèmes qui s'imposent, depuis quelques années surtout, à l'attention des muséographes aussi bien que des savants, des artistes et des éducateurs. Sur ces points fondamentaux, l'accord n'est pas encore fait et ne le sera sans doute jamais car la destination du musée change avec l'homme qui la recherche et avec la génération qui la pose. Et n'en est-il pas ainsi dans tout le domaine où l'homme veut satisfaire à un besoin spirituel? Ne remet-il pas constamment en discussion les buts de l'Etat, du droit, de l'éducation, de l'art, de la science même?

Or, changer de but, c'est changer de forme et de méthode. Mais quel que soit ce but, il demande en tout cas la séparation d'objets qui, par leur nature même, font appel à des instincts et à des penchants différents. Le musée conçu comme institution culturelle, ne peut dès lors conserver pêle-mêle des curiosités, des chefs-d'œuvre de l'art, des objets ethnographiques, des documents botaniques, zoologiques, minéralogiques et des monstres ou anomalies. Il se spécialise. Et même, en admettant que la spécialisation, poussée trop loin, doive céder un jour le pas à une synthèse nouvelle, l'opération se fera désormais selon un point de vue nettement défini et un, sans redonner dans le chaos de jadis.

\section{Les collections ${ }^{4}$}

5 C'est à la suite de cette spécialisation que les instruments de musique, naguère dispersés et logés sans rime ni raison dans les musées, au gré de quelque acquisition de hasard, ont été réunis dans des musées distincts. Le premier pas a été fait par la Convention nationale de France qui, le 3 août 1795, promulgua dans une loi spéciale l'organisation du Conservatoire de Musique et d'un musée d'instruments. Toutefois, ce projet n'a été réalisé que soixante-dix ans plus tard. Entre temps, les collectionneurs particuliers ont inauguré 
cette branche nouvelle : Louis Clapisson à Paris, F.-J. Fétis et Victor-Charles Mahillon à Bruxelles, César Snoeck à Gand, Paul de Wit à Leipzig, et plus récemment Carl Claudius à Copenhague, Mrs. Crosby Brown à New-York, Donaldson et le Rév. Chanoine Francis W. Galpin en Angleterre, Wilhelm Heyer à Cologne, Daniel-François Scheurleer à La Haye, Neupert à Nuremberg, Lecerf à Paris et Schumacher à Lucerne. Parmi ces collections particulières, il y en eut de très importantes, qui, par le nombre et la valeur des objets, et par les soins attentifs de leurs possesseurs, dépassaient de beaucoup le niveau de l'amateur. Ainsi en témoignent des catalogues raisonnés et descriptifs, établis par le collectionneur même ou par un conservateur. Tels sont, pour nous borner à un petit nombre d'exemples, les deux gros volumes de la collection W. Heyer à Cologne (aujourd'hui à Leipzig), rédigés par Georges Kinsky, et les catalogues des collections Claudius, Crosby Brown et Snoeck.

6 À peu près toutes ces collections ont été achetées par des gouvernements d'Etat et transformées en musées publics. C'est ainsi que naquirent, en 1864, par l'achat des 230 instruments de Louis Clapisson, le Musée du Conservatoire National de Musique à Paris ; en 1873, par la cession des collections Fétis et Mahillon, le Musée du Conservatoire Royal de Musique à Bruxelles; en 1888, par l'acquisition de la première collection De Wit, le Musée de la Königliche Hochschule für Musik de Berlin; et, sans poursuivre cette énumération, nous arrivons, en 1929, date à laquelle la collection Wilhelm Heyer, à Cologne, fut achetée en bloc par l'Etat de Saxe et la ville de Leipzig et installée dans cette ville, comme musée public. C'est par une voie semblable qu'un nombre considérable de villes sont arrivées à posséder des musées d'instruments de musique, soit indépendants, soit sous forme de départements distincts de tel ou tel musée. On peut citer en Allemagne: Berlin, Eisenach, Francfort, Hambourg, Leipzig, Markneukirchen, Munich, Nuremberg et Stuttgart; en Amérique : Ann Arbor, Boston et New-York; en Angleterre : Londres; en Autriche: Vienne et Salzbourg; en Belgique: Bruxelles; au Danemark: Copenhague; en France: Paris; en Espagne: Barcelone; en Italie: Florence, Milan et Vérone; en Suède : Stockholm; en Suisse : Bâle; en Tchécoslovaquie : Prague. 
Fig. 1 : Curt Sachs dans la bibliothèques à New York, sans date [début des années 1950]. Photo anonyme, Coll. Florence Gétreau

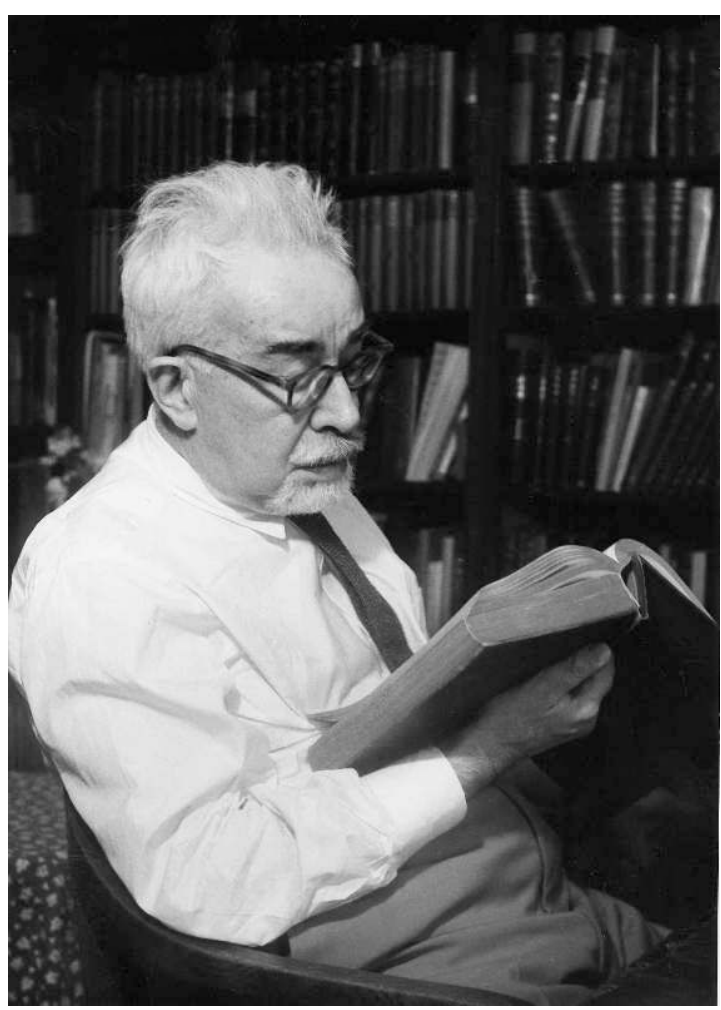

7 Comme tous ces musées doivent leur création à des collections privées, il n'est peut-être pas inutile de s'enquérir des professions de leurs collectionneurs : Fétis était musicologue, Mahillon fabricant d'instruments à vent, Snoeck avocat, De Wit violoncelliste, gambiste et éditeur d'une feuille représentant les intérêts commerciaux de la facture, Heyer et Claudius industriels, Scheurleer banquier; au nombre des contemporains, M. Galpin est chanoine et $\mathrm{M}$. Neupert fabricant de pianos. Ce sont donc là des musicologues, des facteurs, des artistes-éditeurs aussi bien que des amateurs purs. Autant de métiers, autant de points de vue différents. Nous comprenons sans peine l'intérêt que peuvent porter à de telles collections des hommes liés aux instruments de par leur profession. Mais le fait que la plupart de ces collectionneurs ont eu l'idée, voire même la manie de réunir sous leurs yeux et dans leurs appartements des instruments de musique sans être "de la partie ", cela nous laisse entrevoir que le monde des instruments n'attire pas que le seul professionnel.

Il n'est guère facile de préciser la nature de cet attrait particulier. Serait-ce l'union, toute nouvelle, d'objet d'art décoratif et d'appareil technique? Serait-ce la pérennité, non seulement de la forme, mais encore de la voix et, par elle, de l'âme des siècles passés? Tout cela parait coopérer au charme des instruments comme objets de collection. Le tableau, la gravure, la statue, le tapis ravissent l'œil ; l'instrument, par contre, s'adresse à l'œil et à l'oreille: Rendo lieti un tempo gli occhi e il cuore, c'est la devise écrite en lettres dorées sur le devant du plus ancien clavecin italien que possède le musée de Berlin.

De là le caractère particulier et varié qui distingue le musée instrumental de tout autre musée. De là aussi les difficultés considérables que comportent l'administration, la conservation et l'exposition dans une institution de ce genre. Quant à son but particulier, on ne saurait dès à présent le définir : demandons plutôt au conservateur d'un tel musée 
ce que les visiteurs lui réclament. Il vous répondra que son public est des plus mélangé. Ceux qui s'adressent le plus souvent à lui sont des artistes et des musicologues. On ne saurait les séparer, car les plus qualifiés d'entre eux ont des intérêts communs. Leur désir de connaître vise au même but, bien qu'il soit différemment formulé : ils veulent savoir comment "chantent» les instruments d'autrefois et les instruments des peuples étrangers, quel est leur timbre, leur capacité, leur style ; comment il faut interpréter une œuvre de Bach, dont les partitions ne rendent que les signes muets et ambigus, en faisant abstraction de la viva vox; quelle fut la sonorité particulière du XVIIe siècle français, des nuove musiche de Florence, de l'ars nova de Paris; ce que c'est qu'un cornet à bouquin, une régale, une viole d'amour. Puis c'est le facteur qui veut se renseigner: il s'agit de la reconstruction d'un clavecin, d'une basse de viole, d'un orgue de chambre pour l'usage actuel ; quels sont les modèles les plus parfaits ? Il veut apprendre des Anciens, comment ils ont pu donner à leurs instruments, outre la voix, un aspect extérieur si pur, une forme si élégante, une décoration aussi exquise ; il veut voir ce qu'il y a de plus beau dans votre musée. Puis ce sont les artistes, les peintres et les metteurs en scène de théatre ou de film : ils cherchent, pour leurs tableaux ou pour la scène, les spécimens d'instruments, comme ils iront ailleurs chercher des costumes et des armes. Il ne faut pas oublier deux catégories de visiteurs, fort différentes l'une de l'autre. Le connaisseur d'abord, qui s'est spécialisé dans une petite branche, par exemple dans les instruments à archet des environs de 1700 , et qui, sans intérêts historiques ou musicaux prononcés, se plaît à reconnaître et à discuter ces détails infimes invisibles aux profanes, qui font la «main » d'un certain maître. Et enfin, le plus redoutable, l'inventeur qui vous présente le croquis ou le modèle de sa dernière idée, la révélation du secret de Stradivari, la correction qui écartera enfin tous les défauts fâcheux du piston, de cette vis qui transformera le piano d'un seul coup de main en je ne sais quoi. En vain lui parlera-t-on raison, démontrera-t-on que cela a été fait tant de fois sans résultat ni succès, il continuera à poursuivre sa chimère. Nous ne parlerons pas ici des autres visiteurs : classes qui viennent avec leurs professeurs pour trouver au musée une illustration de ce qu'elles ont appris dans les leçons de musique; public sans catégorie particulière, qui s'attache de préférence aux reliques, souvent douteuses, des grands maîtres : clavecin de Jean-Sébastien Bach, violon de Mozart, piano de Liszt, et aux objets de pure curiosité : coussins qui renferment un petit orgue, pochettes contenant à l'intérieur l'archet minuscule et un éventail à l'usage du maitre de danse, aux tables à coudre qui permettent d'interrompre le raccommodage et de jouer une berceuse sur le piano caché dans le tiroir - ce public qui de tout temps nous rappellera que les musées sont nés des cabinets de curiosités des princes et de la commode de la jeune fille de Seldwyla.

10 Le musée d'instruments de musique s'adresse donc à deux sens, à l'ouïe et à l'œil, et chacun d'eux fait valoir des droits tant artistiques que scientifiques. Abstraction faite de la curiosité pure, ce musée présente des objets d'art et des documents techniques ou historiques; et les uns comme les autres demandent a être à la fois vus et écoutés : difficulté considérable, mais pleine d'attraits.

11 Il va sans dire - ou, plus exactement, il devrait aller sans dire - que le sens dominant est l'ouïe. L'instrument n'est-il pas fait pour la musique? Aucun bon facteur ne le crée pour qu'il reste muet et ne constitue qu'une pièce d'art décoratif, et aucune bonne époque n'admettrait qu'il en fût ainsi : c'est là le fait capital. Chacun en conviendra; mais, en réalité, l'état de chose des musées parait confirmer exactement le contraire. Il n'est pas ici question des cas assez rares où l'aspect extérieur d'un instrument fabriqué à 
l'intention de quelque grand personnage, l'emporte à un tel degré sur l'élément sonore, que l'exposition purement plastique dans un musée des arts décoratifs se trouve justifiée. Il s'agit ici plutôt des cas par trop fréquents, pour ne pas dire généraux, où l'instrument même, dépourvu de tout intérêt décoratif, est logé dans quelque musée historique, triste chose sans vie, aux cordes cassées, dont la table de résonance a éclaté, dont les touches pendent. Si vous vous en approchez, le gardien intervient aussitôt : Défense de toucher! Et ne songez pas au privilège que vous donnerait une permission spéciale, car vous entendrez un son par-ci, un son par-là, lambeaux misérables, vides, faux : ce n'est plus que le bégaiement d'un moribond. Pourquoi donc exposer ce moribond? Et n'est-ce pas un crime de le laisser mourir? Ne serait-ce pas, au contraire, le devoir suprême, voire l'unique raison d'être d'un musée que de faire vivre les restes du passé qui lui sont confiés? Le conservateur vous dira que c'est impossible, qu'il n'a ni les connaissances, ni le goût, ni l'expérience, ni les artisans, les outils, l'atelier, l'argent disponibles. Alors, qu'on confie ces instruments aux musées spéciaux!

\section{La restauration}

Aucun musée n'a le droit de posséder des objets qu'il n'est pas en état de conserver.

Cette conservation, il faut le reconnaître, est des plus difficile. Les autres musées ont à nettoyer les nouvelles acquisitions et quelquefois à les désinfecter. Il y aura même des cas où tels objets devront être débarrassés d'adjonctions posthumes. Pour le reste, ce sera uniquement un problème de conservation proprement dite, c'est-à-dire de protection contre les accidents, les mites, l'humidité et les excès de température et de lumière. Aucune de ces obligations n'est épargnée au conservateur d'un musée instrumental, même s'il ne se soucie pas de la vie sonore des instruments. Au contraire, bien souvent, il devra lutter de façon beaucoup plus active contre les détériorations que dans le cas des autres musées. Tout d'abord, les parties vibrantes des instruments sont extrêmement délicates. Puis, plus encore que les objets d'art pur, bon nombre d'instruments ont séjourné pendant des dizaines d'années, voire des siècles, au fond d'un grenier, où les avaient relégués les styles et les modes d'une époque nouvelle ainsi que les perfectionnements techniques survenus depuis lors. Enfin les déformations jouent ici un rôle sans équivalent. Qu'on imagine la carrière d'un instrument quelconque : des mains de son créateur, il passe à celles d'une série indéterminée d'exécutants, qui l'abîment par l'usage constant, et à celles de possesseurs non-musiciens qui, en héritiers insouciants, le ruinent faute d'emploi. L'exécutant, l'accordeur, le réparateur, déforment l'œuvre primitive: les cordes sont renouvelées sans cesse; une table d'harmonie éclatée est simplement recollée; la barre vermoulue d'un violon est remplacée. Il y a plus: tel flûtiste, ayant les mains trop petites, fait fermer les trous trop incommodes de sa flûte et en fait percer d'autres, qui, pour être déplacés, exigent une correction aux dépens de la sonorité ; tel violiste souffre de la tension exagérée du bras que lui impose un alto trop gros pour sa taille, et le luthier se trouve prêt à le rogner. Mais plus graves encore sont les dénaturations que causent les changements de style, et ce sont précisément les instruments les plus précieux qui les subissent le plus fréquemment ; car, pour les autres, on ne prend pas la peine de les transformer, on les met dans la cheminée. Parmi les dénaturations de cet ordre, il faut mentionner le grand ravalement des claviers, conforme à l'étendue croissante du matériel sonore; l'allongement du manche des violons, par suite de l'augmentation du nombre des positions de la main; le renforcement des tables 
d'harmonie pour offrir la résistance nécessaire au diapason élevé, ou bien leur dégagement, pour accroître la résonance; l'addition de pédales qui permettent une variation plus rapide des timbres, l'élargissement de la perce des instruments à vent ; on pourrait allonger la liste indéfiniment.

Dans ces conditions, que faut-il tenter? Devra-t-on renoncer à toute intervention et laisser les objets tels quels ? En Amérique, il est vrai, les musées spéciaux eux-mêmes laissent les instruments exactement dans l'état où ils se trouvaient au moment de l'acquisition : on ne se soucie ni de la restauration de la voix ni de celle du mécanisme ou de la forme extérieure ; aussitôt arrivés, les objets, jusqu'aux clavecins et aux pianos, sont enfermés sous verre. Mais, là encore, on peut se demander une fois de plus à quoi peut bien servir une collection d'instruments de musique si ceux-ci se présentent à la fois muets et faussés?

Mais, objectera-t-on, ne sommes-nous pas enfin sortis de cette fâcheuse époque des remises à neuf? Ne préfère-t-on pas les torses antiques sans bras ni têtes aux tentatives irrespectueuses d'interventions modernes dans le chef-d'œuvre d'un maitre ancien? C'est exact, mais encore faut-il distinguer : les beaux-arts présentent à l'œil ce qui est uniquement destiné à l'œil ; le musée d'instruments de musique, au contraire - nous le répétons - s'adresse et à l'œil et à l'ouïe. Un instrument inaudible est un non-sens presque au même titre que le serait un tableau invisible.

Or, il n'y a guère d'instruments qui aient conservé leur voix sans qu'on ait dû y aider. Car le bois «travaille »; les parties de l'instrument se déforment, les molécules se déplacent sous l'action des différents exécutants, du manque d'usage, de la température, de l'humidité et, ne l'oublions pas, du diapason tantôt haussé, tantôt baissé : cet organisme extrêmement sensible et composé de matières périssables, comment pourrait-il rester intact à travers les siècles? Et nous ne parlons pas même des accidents extérieurs auxquels aucun objet n'échappe, depuis les graves détériorations jusqu'aux petites altérations ou accidents tels que la chute de l'âme d'un violon, qui, ne pouvant être replacée exactement au même endroit, modifie le timbre de l'instrument. Tout cela implique que la raison d'être d'un musée instrumental est étroitement liée à la restauration. Mais quelles en sont les lois et les limites? C'est là qu'interviennent les incertitudes, les doutes, les contradictions. Chaque instrument présentera un cas différent, imposera une décision particulière, exigera un traitement qui lui sera propre.

Il n'est cependant pas inutile de chercher à dégager quelques principes généraux qui pourraient servir de base. Et l'on commencera par se demander ce qu'il ne faut pas faire. 
Fig. 2: Curt Sachs et sa fille Gabrielle, New York, début des années 1940. Photo anonyme, Coll. Florence Gétreau

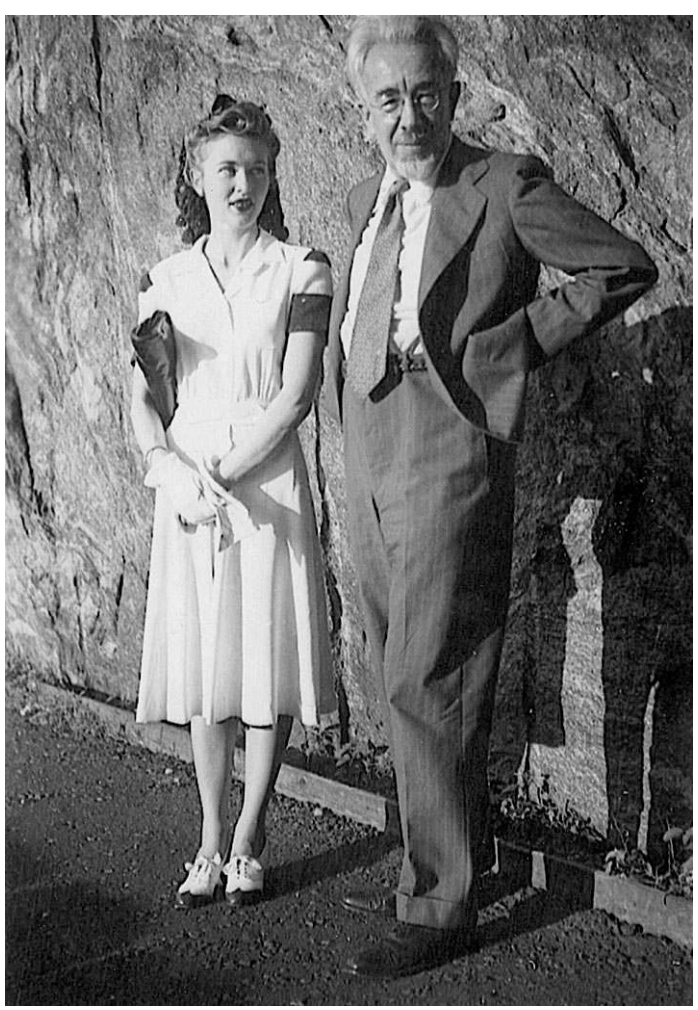

Fig. 3: Cirt Sachs, New York, sans date [début des années 1950]. Photo Joseph Breitenbach, New York, Collection Florence Gétreau

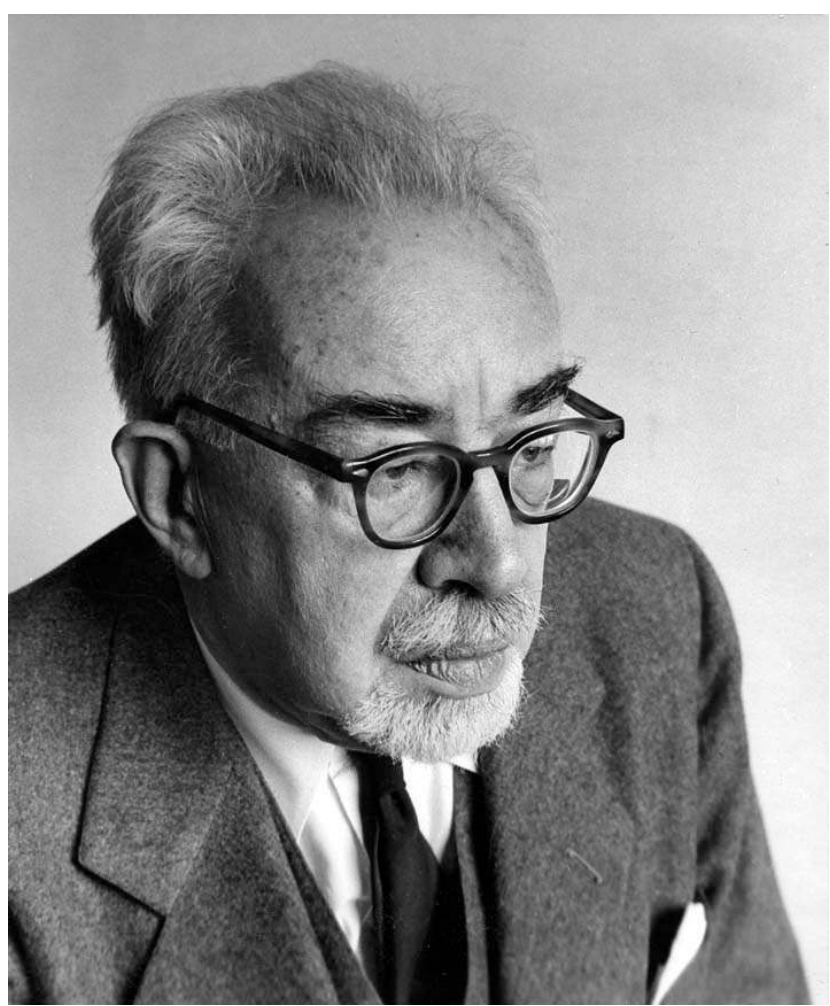


18 À vouloir corriger le maitre ancien et embellir son œuvre, on ne fait que la détruire et fausser le document. N'ajoutons pas un second clavier au clavecin, ne remplaçons pas la tête du violon par une autre, accordée à notre goût personnel, n'augmentons pas le nombre des cordes d'une basse de viole pour en accroître les moyens et la valeur. On objectera qu'il s'agit là du sort commun des instruments de musique, que chaque génération apporte des changements, et que ce serait du pharisaïsme que de l'interdire. Eh bien, non. Ces générations antérieures ont modifié tout naïvement ce qui entrait dans la vie, sans autre intérêt que celui d'adapter les objets aux exigences nouvelles de la vie actuelle. Mais ce que l'on prétend faire aujourd'hui n'a, au contraire, rien à voir avec la vie. Ce n'est pas la continuation et la transformation dans le style du temps actuel, de même que tant de générations fortes et spontanées ont continué et transformé les édifices de leurs ancêtres, mais c'est une petite charlatanerie sans piété, et dont le résultat équivaudra rarement à une tourelle Viollet-le-Duc, de Notre-Dame.

Mais il faut tout autant éviter d'être puristes. Personne ne songera à démolir les parties tardives d'une architecture continuée ou transformée, à moins qu'elles ne soient détériorées, et personne ne se risquera à dégager un clavecin Ruckers du XVII ${ }^{e}$ siècle, de l'agrandissement de son clavier, du "grand ravalement » que le XVIII lui a fait subir, pour l'assimiler aux conditions du moment. La nouvelle forme, comme l'ancienne, est une unité désormais indestructible, et au surplus, elle représente un document historique des plus instructifs ; c'est toute une page d'histoire de la musique qui se déroule devant nos yeux. Le XVIII ${ }^{e}$ siècle est riche en transitions de ce genre. On songera surtout à ces spécimens extrêmement rares qui, tout en gardant du clavecin un rang de sautereaux, ont remplacé l'autre par des martelets du système piano; le heurt entre les époques du clavecin et celle du piano, de la dynamique rigide et de la dynamique sentimentale, ne peut être illustré et conservé d'une manière plus frappante.

20 Citons encore le cas de la basse de viole: exclue de la musique officielle depuis 1750, elle se débarrasse du surplus de ses cordes et partant, du manche trop large ; on l'a munie d'une touche à quatre cordes et elle sert dorénavant comme violoncelle. Se défendra-t-on d'y toucher? Nous ne le croyons guère, car le cas est différent. Tout musée possède une quantité de ces bâtards, il n'y a guère de basse de viole conservée telle quelle. Et après tout, il ne s'agit pas d'une transformation organique de la construction primitive; au contraire, une intervention brutale a détruit le mécanisme délicat de la construction originale, sans respect de l'unité inviolable de la forme particulière et du timbre argentin et svelte, bien que ce qui reste soit entièrement opposé au style violoncelle, et comme forme et comme sonorité. On en laissera intactes une ou deux; mais le reste devra redevenir basses de viole.

21 Si claire que soit cette différence, il se pourra que la pratique et les nécessités muséographiques en décident autrement. Supposons, dans le premier de nos exemples, que la transformation ait été faite par une main extrêmement maladroite et que le clavecin original ait été exécuté par un facteur de grand mérite et nom, ou que le type de ce clavecin soit particulièrement intéressant et non représenté dans le musée, ne faudra$\mathrm{t}$-il pas risquer le dégagement, alors même que la transformation en mi-piano constitue un document historique? Et dans le deuxième exemple: si c'était un maître de grande valeur et de goût qui avait été chargé de la "violoncellisation» d'une basse de viole quelconque, voudra-t-on rejeter son œuvre?

22 Valeur, goût, adresse, maître, intérêt historique, ce sont là autant d'éléments qui conduisent à des considérations, à des doutes, à des restrictions, à des hésitations bien 
compréhensibles. Toute la question semble enveloppée de velléités subjectives. Est-ce à dire qu'il n'y aurait aucune base stable, qui, tout en laissant les décisions dernières au jugement scientifique et artistique du conservateur, lui fournirait néanmoins certains principes? Nous allons tenter d'en établir.

1. La restauration doit rendre à l'instrument la voix.

2. Elle n'aura pas à s'en occuper : $a$ ) si l'appareil sonore est tout à fait perdu ou à peu près, de sorte qu'il ne s'agirait plus d'une restauration musicale, mais plutôt d'une reconstruction plus ou moins libre et sans valeur documentaire. Tel est le cas de beaucoup d'instruments d'ordre décoratif, qui ont été conservés pour la richesse ou l'élégance de leur extérieur; b) si la restauration risque de détruire la valeur archéologique. La harpe égyptienne, par exemple, dont nous avions pu faire l'acquisition pour le musée de Berlin, aurait dû être recouverte d'une peau, dont les traces étaient encore visibles sur le bois. Si l'on avait procédé à cette opération, le bois, c'est-à-dire précisément l'élément parvenu jusqu'à nous, aurait disparu sous la peau moderne, et l'objet, sans reconquérir sa sonorité authentique, aurait été soustrait aux regards. Il était préférable de ne pas toucher à l'original, et d'en faire exécuter un fac-similé mis en état de rendre un son.

3. La restauration de la voix aura à suivre exclusivement et intégralement les données de l'époque en question: le restaurateur s'interdira rigoureusement de corriger l'objet. On commet ordinairement la faute de rechercher un compromis plus ou moins conscient, entre la restauration historique et la restauration pratique. Mais tout essai tendant à adapter l'instrument aux exigences modernes - des concerts de musique ancienne bien entendu -, orchestres forts, salles vastes, diapason de 435 vibrations doubles, timbre sensuel et abondant, tout essai de cet ordre fait tort à la sonorité authentique, et rend illusoire la raison d'être des musées.

4. Il s'ensuit que, non seulement les parties architectoniques, mais encore les cordes et tous les accessoires doivent se conformer minutieusement au style de l'époque originelle. Une corde de piano moderne défigurerait la sonorité caractéristique du clavecin : elle serait trop dure et trop tendue. Le timbre extrêmement délicat, argentin et vibrant de cet instrument, demande des cordes d'une élasticité tout autre. Il est impossible de se les procurer dans le commerce; on devra les faire tréfiler dans une laminerie de cuivre.

5. Les musées disposant d'un certain nombre d'instruments du même type feront, à bon droit, une exception pour avoir des spécimens en état de fournir des voix dans un ensemble composé pour exécuter de la musique ancienne. Ils consacreront à cet usage des exemplaires qui ne sont pas les plus remarquables, et les restaureront de manière à atteindre le diapason élevé en usage aujourd'hui. Mais on n'ira pas plus loin! Ce diapason n'est, du reste, pas toujours contraire aux habitudes des anciens, c'est un préjugé de le croire: beaucoup d'instruments à tonalité stable, bien conservés, de nationalité et d'époque différentes, l'attestent. Quant à la sonorité même, on s'abstiendra strictement de la renforcer ou de la nuancer. Les autres instruments, étrangers au musée et venant du dehors pour être intégrés dans un ensemble, devront suivre l'exemple, car une musique ancienne au timbre moderne, est comme un tableau ancien remanié avec des couleurs du XXe siècle.

6. Dans les cas où l'instrument a subi des transformations avant d'entrer au musée, on aura à distinguer trois données essentielles :

a) Moins une transformation est ancienne, et moins elle présentera d'intérêt muséographique. Tous les musées ont à lutter contre les restaurations grossières du XIXe et même du XXe siècle, personne ne se fera scrupule de les enlever. Plus la date des 
restaurations ou des adjonctions est ancienne, plus elles gagneront en intérêt documentaire. Quelques-uns des cas les plus fréquents ont déjà été cités. C'est d'abord :

b) La transformation conforme au type: le ravalement, par exemple, qui, sans porter atteinte à l'âme du clavecin, n'augmente que l'étendue du clavier; l'apport de clefs additionnelles à une flûte ; la septième corde d'une basse de viole, qui n'en comptait que six.

c) La transformation contraire au type : la base de viole dont on a fait un violoncelle; le luth devenu guitare ; le clavecin aux sautereaux remplacés par des martelets.

Nous en avons dit l'essentiel: les transformations du cas b) seront généralement à respecter; on statuera sur les cas $c$ ) suivant la rareté plus ou moins grande des types intacts. Cela revient à dire que, vu la rareté des basses de viole intactes, on pourra se permettre de reconstruire la basse de viole originale, mais que, par contre, on conservera tel quel le clavecin transformé en piano, parce que ce type, document précieux d'un changement de style, est beaucoup plus rare que les clavecins intégraux.

7. Quant à la restauration des objets eux-mêmes, hormis l'appareil sonore, elle se conformera aux normes valables pour les musées d'arts décoratifs.

Les qualités qu'exige la restauration se trouveront rarement réunies dans la rnême personne. Facteur et luthier, menuisier et tourneur, vitrier et peintre, serrurier et accordeur, le restaurateur doit être doué d'un goût irréprochable et de cette souplesse d'esprit qui permet de pénétrer dans les styles anciens et étrangers. Et toutes ces qualités resteraient insuffisantes, s'il ne s'y ajoutaient la patience, la tranquillité réfléchie, l'amour de l'objet et du détail. En citant, comme véritables restaurateurs, le regretté Frans de Vestibule à Bruxelles, l'aide fidèle de Victor-Charles Mahillon, et MM. Marx et Hartmann, des musées de Leipzig et de Berlin, j'espère et je crains, hélas, de n'en avoir oublié aucun...

\section{L'exposition} nature même des objets. La galerie de peintures réserve aux tableaux les seules parois et abandonne au visiteur l'aire entière pour circuler et stationner ; les musées scientifiques, par exemple les collections minéralogiques, botaniques et zoologiques, peuvent loger la plupart des objets dans des vitrines, soit verticales, soit horizontales, sans barrer le chemin aux curieux ; les musées du mobilier même, forcés d'occuper une partie de l'aire, ont l'avantage particulier que les objets de leurs collections, étant destinés par définition à équiper des locaux, permettent une disposition satisfaisante et un équilibre entre les 
parois et le plancher. En revanche, le musée instrumental est en quelque sorte écrasé par les exigences insatiables des clavecins, pianos et orgues. Supposons qu'une collection ait cent instruments à clavier et que chacun d'eux couvre une superficie de 2 mètres sur 1,50 $\mathrm{m}$. Cela donnerait $300 \mathrm{~m}^{2}$, sans compter l'espace libre nécessaire à la circulation et à l'examen. Si l'on ne réserve à chacun qu'un demi-mètre d'air libre, les cent instruments demandent cent fois la superficie de $3 \mathrm{~m}$ sur $2,50 \mathrm{~m}$, soit $750 \mathrm{~m}^{2}$. Et notons qu'on obtiendrait, à ce régime, une sorte de magasin de cercueils, un entassement ennuyeux des plus lugubre. En réalité on a besoin d'un espace infiniment plus vaste. Mais cela entre dans le domaine de l'installation, qui sera traité plus loin.

Pour le moment il s'agit d'aborder la question fondamentale : quelle sera l'idée dominante de l'installation? Jusqu'ici cette idée, là où, toutefois, elle existe, découle de la spécialisation, et de l'évolutionnisme propres au XIXe siècle : c'est la distribution par types, qui présente séparément, au visiteur, les violes, les flûtes, les trompettes, celles-ci rangées d'après l'ordre chronologique (pour autant qu'on pouvait les dater), chacun de ces groupes étant précédé par quelques prototypes exotiques, voire primitifs, dont ils étaient censés dériver. Quelle satisfaction ne trouvait-on pas à montrer, dans le cadre exigu d'une vitrine, tout le chemin parcouru! Au début, un simple roseau, à peine perforé de deux ou trois trous, et à la fin, une machine surchargée de clés et d'anneaux mobiles : quelle ascension, quelle avance !

Hélas, notre conception de l'histoire n'est plus celle du XIX siècle, et le culte du " progrès ", s'est sensiblement refroidi. Et puis, si le musée a pour but de donner des vues générales, il perd précisément cette raison d'être si on le morcelle en une suite de petits musées de violons, de clarinettes, de pianos.

Si l'on admet que la facture n'a d'autre fin que de servir la sonorité, sans comporter de valeur propre - est-ce une vérité première ? - ce ne sera plus le mécanisme qui, dans les musées, réclamera l'essentiel de notre attention, mais plutôt la sonorité, qui est le but dernier du mécanisme. Nous disons «but», car il ne s'agit pas du résultat fortuit du perfectionnement de l'une ou l'autre branche de la facture: le but est imposé par le besoin, la tendance, le goût des époques et des nations.

31 Nous voici au delà des spécialisations. Notre intérêt ne se bornera pas à constater purement et simplement, que, par exemple, le facteur parisien Sébastien Erard a renforcé l'appareil entier du piano, les cordes, le système des leviers, les martelets, le cadre, la caisse ; on ne se contentera pas d'enregistrer comme un fait quelconque la réforme de la flûte, en 1832, par Théobald Boehm à Munich.

On percevra dans ces deux faits comme des bornes milliaires sur le chemin de cette grande évolution vers la sonorité robuste, qui a ses débuts dans la révolution française, et qui n'est mise en question que de nos jours. Ou, autres exemples: la lutte contre le clavecin, au XVIII siècle, par le piano nouvellement inventé à Florence; la floraison tardive du clavicorde en Allemagne, la guerre des instruments à archet, la victoire de la flûte traversière sur la flûte douce : toutes ces notions n'auraient pas de signification si l'on se bornait à ranger les collections dans des vitrines spéciales, objet par objet, sans communiquer au visiteur l'essentiel. Or l'essentiel, c'est la ruine des grandes hégémonies, de la monarchie absolue, de l'église orthodoxe ou plutôt dogmatique et militante, et l'avènement de l'époque bourgeoise et piétiste, sentimentale et rationaliste. Ni la peinture ni l'architecture de l'époque ne sont des miroirs aussi fidèles de cette transition que la musique. Une telle évolution se révèle en effet très nettement dans le contraste entre les instruments anciens, d'une part, - dont l'expression dynamique est et veut être 
restreinte, - et les nouveaux venus d'autre part, avec leur forte et piano, avec les nuances infinies du crescendo et du decrescendo, avec la faculté illimitée d'exprimer les sentiments individuels de l'homme, ses passions et ses rêves. Il sera superflu de multiplier les exemples, de parler de la transparence du timbre de la Renaissance, ou de la différence des sonorités allemande, anglaise, flamande, française, italienne. Dans les cadres de notre étude, quelques indications suffiront: l'organologie, la science des instruments de musique, fait partie de la musicologie, et la musicologie n'est qu'une branche de l'histoire de la civilisation. Si l'on élève le musée instrumental à ce niveau, on élargit du même coup sa portée, et cela dans un domaine de première importance. Considéré sous cet angle, l'instrument de musique cessera d'être l'affaire des seuls amateurs, facteurs, musiciens et musicologues ; intimement lié aux évolutions générales familières à tous, il entrera dans le domaine de tout érudit, quelle que soit sa spécialité.

Le but ainsi défini, il s'agit d'étudier les moyens de l'atteindre. Les petites collections n'ont pas le choix : elles exposeront ce qu'elles possèdent. Les grandes, en revanche, se garderont bien de tomber dans l'erreur ancienne qui consiste à vouloir tout montrer, erreur qui fatigue le visiteur et qui entrave la clarté de la disposition. On a abondamment discuté, dans ces dernières dizaines d'années, le pour et le contre de la scission des musées en deux parties. Nous ne faisons pas ici allusion à la division en musée-exposition et musée-magasin, - elle est toute naturelle et communément acceptée pour toutes sortes de musées. Le système auquel nous songeons consiste à décomposer le musée en une partie destinée au visiteur, qui recherche une impression générale, un résumé, un enrichissement, collection publique (Schausammlung), et en une autre partie destinée aux études spéciales, collection d'études (Studiensammlung). Cette solution n'est pas idéale pour les musées d'art pur, elle ne semble guère pouvoir être écartée pour les musées miartistiques mi-scientifiques. D'un côté, l'étudiant a besoin d'une documentation aussi complète que possible ; d'autre part, le visiteur ordinaire a le droit de pouvoir contempler un choix des pièces les plus remarquables, soit comme documents historiques, soit comme chefs-d'œuvre. Ces deux qualités - document et chef-d'œuvre - sont bien différentes et souvent même contradictoires. Car le document vise, non pas à la perfection, à la production hors pair, mais plutôt à ce qui peut être considéré comme l'état normal, typique d'une certaine époque ou nation. Il ne saurait donc être question d'une Tribuna florentine des pièces rarissimes, sorte de pot-pourri, dans lequel, pour prendre une image, les mélodies célèbres, détachées de leur ambiance et privées de leurs voisins de partition moins parfaites, s'entassent jusqu'à se détruire réciproquement. Au contraire, le visiteur aura à orienter son admiration vers les cimelia, à partir du niveau ordinaire.

\section{L'instrument}

Voici donc comment s'esquisserait une collection publique ainsi conçue. Le visiteur entre dans la salle des primitifs et de l'époque préhistorique. Il ne se trouvera pas devant un amas de bizarreries poussiéreuses appliquées à la file sur la paroi ou logées dans les vitrines, et classées sur la base d'une évolution de facture, en général plutôt supposée que prouvée. Il ne s'agit guère ni du potentiel, de la capacité de l'instrument ni des progrès de la facture. Car avant que la musique ne devienne un «art » - au sens restreint d'une jouissance, d'une distraction ou d'une édification - elle repose indissolublement dans la totalité psychique de l'homme : elle est action religieuse et sociale et, par là, le miroir 
fidèle qui reflète les traits des civilisations anciennes. L'évolution des instruments de musique, commence par une impulsion motrice, l'impulsion innée de frapper, secouer, souffler, gratter, pincer, frotter, l'impulsion qui conduit à s'efforcer ou à se contenir, à s'épancher ou à se replier sur soi-même. Mais cette impulsion n'est pas arbitraire. Elle dépend de la constitution variable des peuples, de cette même constitution qui donne le jour à toutes les idées religieuses de telle ou telle civilisation, à tout ce monde bizarre des cultes et des rites. Les cultes, somme toute, n'ont qu'un seul but: de sauver la vie humaine par la nourriture, la guérison, le rajeunissement, la procréation, la naissance, la transmission et le retour. Les moyens rituels cependant diffèrent : il en est de visuels, de tactiles, d'olfactifs et d'acoustiques. Dans le nombre, le moyen le plus vif et personnel, le plus immatériel et métaphysique, c'est le son. Par conséquent, l'agent sonore, l'instrument de musique, se trouve constituer le charme le plus efficace. Il agit immédiatement, il répond à des mouvements précis. Les autres charmes ont besoin d'une spiritualisation ; l'instrument de musique est esprit. C'est pour cela qu'il occupe le centre de toute vie religieuse. En tant que charme, l'instrument de musique exclut toute conception purement esthétique. Il veut agir - non pas en donnant des jouissances artistiques, mais en suscitant les forces conservatrices et en bannissant les forces destructives. Ainsi l'instrument se forme de prime abord d'après les idées religieuses et cosmogoniques de sa civilisation. Mais l'échange pacifique ou belliqueux, les migrations des peuples et les conquêtes détruisent les idoles de naguère; les religions et les idées cosmogoniques se transforment, changent, disparaissent. Avec elles, les objets du culte qu'on leur voue perdent leur sainteté, leur puissance magique, leur force intrinsèque. D'âme qu'ils étaient, ils deviennent corps. Ce n'est que dans cette période de profanation qu'ils se vulgarisent et sont voués à l'usage pratique de tous les jours : les trompettes ne servent plus au culte solaire et leur fonction s'abaisse jusqu'à donner des signaux de village à village; les tambours perdent le symbolisme féminin et transmettent les messages à travers les forêts vierges, les planchettes ronflantes cessent de représenter les ancêtres et ne servent plus qu'à chasser les éléphants envahissant les plantations; les flûtes, ne pouvant plus enchanter, se mettent à chanter. Et ce n'est qu'à cette époque de profanation que les principes musicaux et techniques viennent jouer un rôle dans la facture. On amplifie dès lors la force sonore, on améliore le timbre, corrige l'échelle, enrichit les moyens, facilite le maniement. On accorde les peaux des tambours, le nombre des cordes s'accroit, et les flûtes adoptent successivement les trous latéraux.

C'est là toute une préhistoire musicale que la salle d'entrée devrait mettre sous les yeux du visiteur. Car même dans les temps historiques, l'émancipation vis-à-vis de ce monde extra-musical n'a jamais été complète. La chaîne qui lie la musique à la métaphysique n'est pas rompue: les idées changent, mais elle s'assurent toujours à nouveau une influence décisive sur la musique.

Certes, il n'est pas facile de faire défiler cette évolution devant les yeux du visiteur. Mais n'aurait-elle pas plus d'intérêt que l'autre évolution, un peu banale, et qui concerne le développement mécanique des différentes familles et espèces d'instruments? Le plan de la préhistoire des instruments a été retracé dans un ouvrage que nous avons publié en $1929^{5}$ et où l'on trouvera les conceptions et les idées qui ont agi sur la main et l'outil, pour former et pour transformer les instruments de musique. Le musée pourra illustrer ces notions en s'inspirant par exemple de l'ordre des chapitres de cet ouvrage, pour ranger les objets. De la sorte on verra d'un côté les timbres stridents, aigus et beuglants, les formes sveltes et aiguës, les rhombes, les trompettes, les flûtes et leurs émules, qui seront 
en quelque sorte les représentants sonores de cette moitié de l'humanité, dont les idées religieuses, cosmogoniques et sociales sont issues d'un caractère viril, chasseur, guerrier, patriarcal, et qu'on peut nommer - sinon très correctement, du moins de façon brève et intelligible, - la moitié totémique. De l'autre côté on verra les tambours et les instruments à cordes, les formes rondes, creuses, sans arêtes ni angles, les timbres sourds, sombres et creux comme symboles sonores des civilisations plutôt féminines, patientes, constructives, qui donneront le jour à l'agriculture et aux formes sociales qu'elle entraîne. Il ne sera pas difficile de mettre en valeur le symbolisme de la forme (et quelquefois même de la couleur); des étiquettes, pas trop nombreuses et pas trop rares non plus, guideront le visiteur. Mais on rédigera les notices ou étiquettes de la façon la plus concise possible: le visiteur est venu pour voir et non pas pour lire. L'élément acoustique fera l'objet de visites guidées.

Les salles des hautes civilisations de l'Orient et de l'Antiquité occidentale montreront le confluent de ces courants, l'affaiblissement du symbolisme magique et, au cours de la profanation progressive, l'évolution de l'importance esthétique et technique. En ExtrêmeOrient, aux Indes, dans les civilisations anciennes du Proche-Orient et de la Méditerranée, la séparation entre un passé magico-symbolique d'un côté et un avenir musico-artistique de l'autre, peut être facilement représentée par le contraste des instruments du culte et de ceux qui servent aux amusements profanes. A cet égard, on insistera principalement sur la transformation continue des instruments à bruit en instruments de musique capables de chant mélodique.

L'antiquité, il est vrai, nous a laissé peu d'instruments ; la terre de Grèce n'est pas propre à conserver les objets de matières périssables, et pour ce qui est de l'Egypte et du ProcheOrient, la plupart des instruments que révèlent les fouilles, resteront aux musées archéologiques, en relation avec les missions. Le musée d'instruments est, pour cette époque là, forcément tributaire des moulages et des reproductions photographiques; mais le conservateur, à moins qu'il n'ait les connaissances archéologiques nécessaires, devra recourir aux avis éclairés d'un bon archéologue de métier, pour ne pas être victime des nombreuses falsifications dues à la restauration tardive de statues antiques. Remarquons que ce sont précisément les instruments de musique qui, étant des parties saillantes de la statue, ont été particulièrement endommagés et, par suite, restaurés.

Malheureusement les instruments originaux du moyen âge nous manquent également. A part quelques fragments de harpes et un petit nombre de trompes, les siècles antérieurs au XVI ${ }^{e}$ 'ont laissé aucune trace instrumentale. Et cette lacune est d'autant plus sensible, que c'est précisément l'époque à laquelle la musique européenne subit la crise d'une influence décisive de la part de l'Orient et d'une tendance progressive vers un langage musical particulièrement occidental.

40 Cependant, l'influence orientale a été, pendant de longs siècles, si forte et si profonde qu'il faudra placer ici, comme documents suppléants, les instruments du Proche-Orient, notamment de l'Afrique blanche. D'un côté, si l'on fait abstraction des quelques modifications apportées tout récemment à ces instruments, on peut être à peu près sûr qu'il n'ont pas changé depuis le moyen âge. D'autre part, il suffit d'examiner les peintures espagnoles, françaises et italiennes du moyen âge, pour voir combien la musique européenne de cette époque s'est servie de la lutherie orientale. Les noms mêmes des instruments médiévaux le démontrent: le rebec n'est autre chose que le rebâb des Arabes, le luth doit son nom - comme sa forme - à al 'ûd, le caño au kanûn, le kobus au kobûz, l' 
ajabeba à al-sabbâba, la gaita à la gaida, l'anafil à al-nafir, les nacaires aux nakkarât, le tambour au tambûr.

Mais les instruments purement orientaux ne seront pas seuls à représenter le moyen âge européen. On en trouve des traces encore plus directes dans la musique populaire de l'Europe actuelle. Si l'on expose la dulzaine et le caramillo de l'Espagne, avec leurs timbres aigus et stridents, le piffero de l'Italie méridionale, le galoubet et les tambourins du Midi de la France, les cornemuses de toutes les régions de notre continent, bref, l'héritage entier des ancêtres que les paysans ont pieusement conservé, on aura une vue d'ensemble du Moyen Age.

Les principes que l'on peut suivre dans les salles des temps modernes, ont été esquissés plus haut. Du moment que c'est l'action des différents âges plutôt que celle des nations, qui a formé les grands styles, on ne classera pas en Allemagne, Angleterre, France, Italie, Pays-Bas, mais par époques. Non pas par siècles : car ces divisions coïncident rarement avec les stades évolutifs. On préférera distinguer, d'abord, jusque vers la fin du XVIe siècle, l'époque Renaissance, caractérisée par un timbre net et transparent et par une sonorité, riche en contrastes il est vrai, mais contenue et éloignée de tout expressionnisme sentimental. On aura ensuite le complexe du style baroque, depuis la fin du XVIe siècle jusqu'à 1750 environ, année de la mort de Jean-Sébastien Bach : c'est la dramatisation du timbre et des moyens dynamiques, qui garde cependant, dans l'expression, la base non-dynamique. Vient ensuite le style du rationalisme: sobre, du piétisme religieux, de la sentimentalité bourgeoise, jusqu'à la Révolution française. Depuis, en 1790 à peu près, on perçoit les débuts de cette sonorité forte, cuivrée et quelquefois grossière, du XIX ${ }^{e}$ siècle, dont le $\mathrm{XX}^{\mathrm{e}}$ bat la chamade.

Tel est le cadre. Pour qui est versé dans l'histoire des instruments et de la musique, il sera facile de le remplir. Toute transformation, toute création nouvelle, la moindre correction technique témoignent d'une évolution ou d'une révolution stylistique. L'évasement du pavillon des trompettes et trombones, au début du XVII e siècle, illustra la sonorité croissante du style baroque; les contrebasses et les contrebassons de cette époque refléteront la tendance vers les couleurs opaques et foncées; les perfectionnements des pistons et des cylindres auront à exprimer toute une histoire du changement d'état " social » des trompettes, jadis " chevaliers " privilégiés, éloignés du contact immédiat avec les "musiciens ", mais, depuis le XVIII ${ }^{e}$ siècle, dépossédés de tout monopole, insérés dans l'orchestre et soumis à une égalité musicale et technique vis-à-vis des autres exécutants. En disposant ces témoins, on s'efforcera de dégager des ensembles caractéristiques pour chaque époque et, dans l'époque, pour chaque pays. La salle de la Renaissance mettra en relief - notamment dans la section allemande - les douzaines de types d'instruments en bois qui font l'orgueil des Stadtpfeifereien: les bombardes, cromornes , cervelas, musettes, schriary, sourdons. Le style baroque, qui a chassé ces timbres multicolores et rigides, tend vers une certaine monochromie, comme le fait, du reste, la peinture contemporaine : les instruments à cordes, et particulièrement ceux à archet, viennent au premier plan et en arrivent à former le noyau des musiques d'orchestre et de chambre. Ce seront eux, ce seront les familles des violes et des violons, qui occuperont la place privilégiée de cette salle. Depuis la fin du XVIII ${ }^{e}$, l'orchestre est fortement nourri par la musique militaire, et les inventeurs les plus ingénieux - il suffit de citer Adolphe Sax à Paris - se sont voués au perfectionnement des instruments à vent : les bois et les cuivres se placeront au centre de la salle du XIX ${ }^{e}$ siècle. Ces groupements, indispensables pour l'orientation du visiteur, permettront en même temps d'éviter un danger auquel se 
heurte la collection d'exposition: en cherchant uniquement à "documenter" par le moyen de telle ou telle pièce remarquable, en la retirant de l'ambiance de ses semblables, on s'expose, pour ainsi dire, à créer un organisme anémique.

\section{L'installation}

Pour plus de clarté, il est nécessaire de préciser le plan d'une salle quelconque, la salle baroque par exemple. Il y a deux dispositions que nous ne pouvons approuver: la première serait la disposition généralement appliquée dans les collections, tant privées que publiques, et qui consiste à exposer plus ou moins tous Ies objets qui rentrent dans ce cadre, à condition qu'ils ne soient pas par trop abîmés ou défigurés par des restaurations. La seconde disposition, également à déconseiller, comporterait pour ainsi dire, une série des types construits et utilisés à cette époque, une collection d'échantillons représentatifs. Cette collection serait forcément statique; elle isolerait les objets au lieu de les relier; elle couperait les styles au lieu de montrer la courbe croissante et décroissante, les tendances de la facture, du goût, de la mode, enfin, l'élément dynamique de la vie musicale.

À l'encontre de ces systèmes de présentation, nous préférerons nous en tenir aux phénomènes caractéristiques principaux. Ce sont, sous l'angle de la sonorité, la basse continue, c'est-à-dire l'accompagnement obligatoire par accords, des instruments à clavier et de leurs pareils; la tendance vers les timbres foncés, le monopole presque absolu des instruments à cordes et la lutte entre les dynamiques glissante et graduée (par «terrasses »). Le phénomène de la basse continue demande à être illustré par un groupe représentatif des instruments à clavier. L'Allemagne, l'Angleterre, la France, l'Italie ont contribué à leur perfectionnement, mais le centre de la fabrication fut la partie flamande des Pays-Bas, notamment Anvers, et parmi les facteurs d'Anvers la célèbre famille des Ruckers. Aucun conservateur ne se privera d'exposer précisément les plus belles pièces belles à la fois comme meubles et comme agents sonores. Cependant il aura à exposer également l'un ou l'autre des clavecins médiocres des Ruckers, puisqu'ils témoignent d'une industrie à grande échelle, d'une vulgarisation tout à fait remarquable, des clavecins comme instruments de la vie quotidienne. Ce groupe, bien composé, donnera en même temps une idée des efforts que la facture a faits pour adapter le clavecin à ses tâches nouvelles. Il montrera la duplication du clavier, qui permet, et dans le solo et dans l'accompagnement, de changer immédiatement la sonorité; il montrera le nombre croissant des registres et leur maniement de plus en plus pratique, et en même temps la disparition complète des épinettes à un seul registre: illustration particulièrement saisissante de la tendance vers l'enrichissement des timbres. Les clavecins, utilisés comme instruments de l'accompagnement par accords, sont, dans ce style normalement aidés par des théorbes ou des chitarroni: on les exposera donc dans leur voisinage, pour souligner leur corrélation. La basse elle même, qui constitue la substruction de cet édifice harmonique, étant trop faible sur les clavecins et les luths, est généralement renforcée par un basson, un violoncelle ou une contrebasse. En même temps, ces instruments correspondent au goût des couleurs foncées et de l'allure pesante de l'époque: on ne reléguera donc pas, comme on en a l'habitude, les contrebasses dans les emplacements les moins favorisés. Nous arrivons maintenant aux instruments à archet, le groupe privilégié du siècle. Ici encore, on ne se bornera pas à un ou deux spécimens, mais on représentera, par le nombre, la richesse des types, les variations de la facture, l'importance de cette 
branche de la lutherie. Il s'agira de faire revivre plastiquement l'épanouissement des deux familles, des viole da gamba (basses de viole) et des viole da braccio (violons), leur guerre de cent ans, les essais tardifs de sauvetage des viole da gamba par la création des violes d'amour et des barytons, et la victoire définitive du violon et de ses congénères, correspondant, mieux que les basses de viole, aux exigences dynamiques et expressives du XVIII ${ }^{e}$ siècle.

On touche dès lors à un point délicat. Montrer l'épanouissement du violon, de l'alto et du violoncelle, n'est-ce pas du même coup attester l'œuvre immortelle des Gasparo da Sal-, Maggini, Amati, Stradivari Guarneri, Bergonzi, des Stainer et Lupot? Mais peut-on les exposer et, si c'est le cas, est-il opportun de les exposer? Il y a deux moyens d'acquérir : l'achat et le don. Vu les prix énormes de ces pièces - prix qui dépassent souvent et de beaucoup le million - les musées n'auront pas la possibilité d'en faire l'acquisition. Reste le don. Mais dans cette catégorie de valeur, il n'y a guère de cadeau ou de legs qui soient faits sans conditions, et la plus regrettable en même temps que la plus fréquente exige que le violon reste tel quel et stipule que, le maitre qui en fut le possesseur étant mort, plus personne ne jouera sur cet instrument. Cette soi-disant piété mêlée de fausse sentimentalité et même d'égoïsme, immobilise ainsi un organisme sensible, créé pour chanter et capable de donner de la joie et des émotions élevées aux générations successives! Cette barbarie ne rappelle-t-elle pas la crémation des veuves hindoues, qui ne doivent pas survivre à leur mari ? Hélas, le fameux violon de Paganini qu'on vous permet d'admirer de loin, abrité sous sa cloche au musée de Gênes, n'est pas seul à être condamné au mutisme.

Or, à notre avis, un instrument appartenant à la vie musicale contemporaine, n'a rien à faire dans un musée, à moins qu'il ne soit plutôt type qu'individu. Ce n'est pas à dire qu'on doive exclure une section moderne dans le musée : tous les objets peuvent être retirés de la vie, du travail, tant qu'ils sont remplaçables; le fabricant en a d'autres. Or le Stradivari qui est encore plein de vigueur, le Guarneri qui n'a pas perdu sa voix, sont des individus qu'on ne peut pas remplacer. Ils ont le droit de vivre selon leur destination, d'être entre les mains d'un artiste de qualité, d'être employés et écoutés.

Et la documentation du musée ? dira-t-on. Le musée étant un miroir de la vie, et non pas, inversement, la vie le miroir d'un musée, il n'y a pas d'hésitation à avoir. Le triomphe du violon dans l'évolution que nous venons de retracer, peut être tout aussi bien documenté par des pièces de valeur moindre. Le musée, il est vrai, ne serait pas complet s'il ne se souciait pas des grands maîtres et de leur œuvre. Mais il pourra se borner aux moulages, aux photos en grandeur naturelle et aux échantillons de vernis. Mais ce matériel sera destiné à la collection d'études, dont il sera question plus loin.

Cependant, on pourra se demander dans quelle mesure il faut admettre, dans les salles d'exposition publique, les photographies ou, plus généralement, les illustrations: peintures, gravures, estampes, dessins, sculptures, voire maquettes. On peut y recourir à trois points de vue différents. D'abord sous l'angle de la documentation technique. Les tendances historiques ne doivent pas nous faIre oublier que le visiteur - et même celui qui parcourt les salles à la hâte - a le droit de savoir ce que dissimule l'enveloppe extérieure des instruments et comment ils sont construits. On aura à exposer, à côté des objets réels, les coupes révélant l'intérieur et le fonctionnement du mécanisme. La grande différence, par exemple, entre les divers instruments à bois du XVI ${ }^{\mathrm{e}}$ siècle, les cromornes, les bombardes, les cervelas, etc., est presque invisible sur les instruments mêmes ; ce n'est que la coupe qui montrera le degré d'élargissement et de conicité. D'autre part, un 
développement comme celui des pistons de l'instrument à cuivre, au début du XIX ${ }^{\mathrm{e}}$ siècle, reste incompris si l'on n'expose des graphiques techniques. Les modèles démontables en seront le complément naturel. Au Musée de Berlin, on a retiré et placé sur le couvercle la dernière touche de chaque instrument à clavier, avec le mécanisme complet des sautereaux ou des martelets. D'autre part, on a scié en deux des têtes de flûtes, des pistons et des cylindres de cornets. L'orgue, dont la force et la variabilité forment un contraste si prodigieux avec le jeu tranquille de l'organiste, demande même des modèles spéciaux et réduits, pour laisser voir un peu cet entassement de soufflets, de sommiers, de soupapes, de tuyaux, de conduits pneumatiques et de contacts électriques.

\section{L'iconographie}

Le deuxième point de vue qui commande l'illustration est celui de l'iconographie proprement dite. Ici, l'illustration remplit une tâche des plus importantes : l'instrument qu'on expose montrera sa forme, et peut-être son mécanisme et ses moyens musicaux. Il n'énonce, par conséquent, qu'une demi-vérité, dangereuse comme toutes les demivérités : il ne laisse percevoir que ce que nous autres modernes, sommes à même d'en retirer, avec nos habitudes, notre technique, notre goût. C'est en revanche la gravure du temps qui nous renseigne sur cette différence profonde, inconnue à la plupart des musiciens et même des musicologues, entre le jeu d'aujourd'hui et de jadis, entre les attitudes de l'exécutant, les positions des mains, des doigts, de l'archet, différence qui pourtant détermine le timbre, le style, bref, la musique. Cette iconographie nous apprend surtout la manière extrêmement délicate dont on tenait et conduisait l'archet : quiconque connaît cette manière, sait que l'on détruit le caractère propre de !a musique ancienne en lui imposant la vigueur du coup d'archet moderne.

51 Si les gravures, dessins et estampes sont indispensables à ce point de vue-là, elles ont, par surcroît, une mission esthétique : une collection d'instruments est composée presque exclusivement de bois; et le bois n'est que rarement peint. À part quelques objets en métal luisant, d'ivoire et de bois peint, le visiteur ne voit que du brun; il sera vite fatigué si l'on ne réussit pas à lui offrir une certaine variété. Selon notre expérience, il y a peu de moyens plus sûrs que les illustrations et, avant tout, la gravure, qui, par son fond blanc, par sa planéité et par son échelle différente, repose l'œil en lui fournissant un contraste.

Toute cette question extrêmement importante est malheureusement négligée dans les musées instrumentaux. Le conservateur, généralement musicien ou du moins versé dans la musique, n'a que trop souvent l'œil insensible. Il estime qu'il ne s'agit ici ni de tableaux ni d'art décoratif, et que lorsqu'on est entre musiciens théoriciens ou artisans, on n'a que faire d'un étalage impressionnant et artistique : il suffit de voir et d'entendre.

Pareille attitude est regrettable, mais encore faut-il se mettre d'accord sur ce qu'on entend par exposition esthétique. Nous ne parlons pas ici de ce que le tapissierdécorateur de la fin du XIX ${ }^{\mathrm{e}}$ siècle comprenait par le mot " artistique ». Nous ne parlons ni de ces trompettes, plaquées en éventail sur la paroi ni de ces bouquets-trophées, qui mettaient à contribution toutes les catégories d'instruments. L'« esthétique » de notre étalage peut être résumée en très peu de mots. Chaque musée du monde fait appel à l'attention active du visiteur et, en même temps, quelles que soient l'importance et la diversité des objets de ses collections, il risque de nuire à cette attention, par excès de tension et par la surabondance des spécimens. Il appartient donc à la disposition et à l'étalage d'obvier à ce risque et d'éveiller, de maintenir et de stimuler l'attention. On 
atteindra le but en trouvant le juste milieu entre la surexcitation et l'ennui. Ce juste milieu cependant ne saurait être calculé; le goût seul le saisira, et sans ce goût, le directeur, même d'un musée instrumental, restera imparfait.

Il est difficile de tracer en quelques lignes le plan d'un étalage idéal, qui dépendra toujours des locaux, de l'importance du musée et des objets mêmes. Mais les principes se dégagent clairement, comme on le verra dans ce qui va suivre.

\section{La scénographie}

La mise en scène a deux buts : faire valoir les pièces principales et mettre le visiteur à son aise en lui épargnant la fatigue inutile. Le premier de ces buts devrait aller de soi si on ne le sacrifiait pas toujours à la manie des séries évolutives. L'objet, rare ou unique par le nom de son créateur ou de celui qui en a joué, par son type ou sa facture, doit être placé à part, en dehors de la ligne générale, habituellement dans sa propre vitrine. Mais il faut se garder de renfermer dans des vitrines les instruments à clavier, comme on le fait en Amérique. Les instruments à clavier sont des meubles, destinés à vivre avec nous, dans le même espace. Les reléguer derrière une vitre, c'est rappeler un peu la préparation anatomique ou le jardin zoologique; à voir, dans quelques musées d'outre-mer, les pianos-girafes tendre le cou au-dessus de la cloison, il est difficile de ne pas songer à l'homonyme quadrupède du jardin d'acclimatation. Si l'étalage doit servir à mettre le visiteur à son aise, on conviendra qu'on n'atteint pas ce but en séquestrant chaque objet : le visiteur se sent étranger, exclu, repoussé. Tout ce qu'un musicien soigneux serre, après l'usage, a sa place dans les vitrines; en revanche, les instruments-meubles doivent être librement exposés. Si l'on veut traiter l'un d'entre eux avec plus d'égard, il suffit de le mettre sur un socle ; pour lui épargner le jeu indiscret des profanes, on pourra protéger son clavier par un couvercle vitré.

Les autres pièces principales du musée auront leurs vitrines à elles, montées sur un socle. La hauteur du socle - et nous touchons ici à un problème qui concerne également le second but de la mise en scène - se règle en générai sur la nature de l'objet: celui-ci présentera la déformation minima de perspective et il causera au visiteur le minimum de fatigue, si son milieu est à quelques centimètres au-dessous de la hauteur moyenne des yeux, c'est-à-dire à 140-150 cm environ. Ce principe, il est vrai, ne sera pas toujours réalisable; notamment les petits objets, comme les violons, demanderont, pour des raisons d'espace, une disposition en deux ou trois rangs l'un au-dessus de l'autre. En ce cas on s'expose à un raccourci fâcheux des objets du premier et du troisième rang, qui nuit à la finesse mesurée des proportions, tout en causant au visiteur une certaine gêne dont il ignore le pourquoi. Pour remédier à cet inconvénient, on relèvera légèrement, par un appui invisible quelconque, la partie critique ; soit l'extrémité supérieure dans le rang supérieur et l'extrémité inférieure dans le rang inférieur. Toutefois, même en procédant à cette légère correction, on évitera de placer les objets en dehors d'une zone visuelle assez limitée, dans le musée instrumental comme dans tout autre musée. Ce qui dépasse $50 \mathrm{~cm}$ vers le bas et $2 \mathrm{~m}$ vers le haut, demande au visiteur un effort fatigant et généralement inutile.

Dans une même vitrine, il n'est guère recommandable de mêler les formats: l'œil et l'attention auraient à s'adapter différemment - un violon et une contrebasse se nuisent réciproquement. Il faut surtout que la troisième dimension soit à peu près égale ; si l'on applique Ies instruments au fond de la vitrine, et l'on y sera forcé dans la plupart des cas, 
Ie mélange, par exemple, des violes plates et profondes, est désagréable à l'œil. Pour éviter ces inconvénients, nous avons ménagé, dans les vitrines du Musée de Berlin, deux ou trois étages de hauteur et de profondeur différentes. La vitrine des altos et des violoncelles, par exemple, comporte un socle appuyé contre le fond et sur la face duquel sont appliqués les altos, tandis que les violoncelles restent debout en dessus; dans la vitrine des anches doubles, nous avons disposé d'une manière analogue les bassons en dessus, sur le socle, et les hautbois, en bas, contre le socle. On confère ainsi un rythme très simple à la vitrine, et les différents formats et profondeurs ne se gênent plus réciproquement. Suivant les dimensions de la vitrine, il pourra être indiqué d'interrompre le socle ou de le graduer, pour ne pas avoir la monotonie lassante des horizontales continues. En le faisant saillir plus ou moins, on conférera à la vitrine une certaine articulation rythmique, dont on peut profiter pour séparer les différents groupes d'objets.

Dans la vitrine, on placera les instruments dans leur position naturelle (qui n'est pas toujours celle qu'on leur donne pendant le jeu). Certes, il est de beaucoup plus commode de poser un ophicléide, un tuba contrebasse, un saxhorn, pavillon en bas, sur le socle, plutôt que de les suspendre, pavillon en haut, moyennant toutes les tracasseries que causent le poids, l'évasement du pavillon, la complication et l'asymétrie des tuyaux. Mais rien n'est plus fâcheux. Car tout instrument bien fait est un organisme d'une conception presque anthropomorphique: ce n'est pas une métaphore vide de sens que de parler de la patte d'un hautbois, du dos d'un luth, de la tête d'un violon et - du moins en langue allemande - de son cou (Hals) et de sa poitrine (Brust). Il est impossible de négliger cette architecture naturelle et organique de l'instrument; si l'on voulait la négliger, on donnerait dans le genre magasin ou dépôt, plutôt que musée. La suspension pose également un problème : on ne saurait se contenter de planter un clou dans le fond et d'y suspendre l'objet en l'abandonnant au hasard de son déséquilibre; il donnerait l'impression misérable du pendu au gibet ou du bœuf à la boucherie, mais jamais celle d'un organisme vivant. Si l'on veut éviter l'impression désagréable d'une boutique de bric-à-brac, de poussière et d'abandon, il faudra se conformer à la règle selon laquelle pour tout instrument - les instruments à clavier exceptés - l'axe central doit rester, pour l'œil, strictement vertical ; qu'il ne cède ni à droite ni à gauche, qu'il ne penche ni en avant ni en arrière. Vertical pour l'œil, s'entend; car, nous en avons parlé ci-dessus, il faudra, pour ne pas risquer le raccourci de perspective, adapter à la parallaxe du regard, les objets placés trop haut ou trop bas.

59 La tendance moderne a opté pour la toile grise ou jaunâtre comme fond des vitrines. De fait, ce timbre calme et neutre est excellent et s'accorde bien avec le bois brun de la plupart des instruments. A la longue, il est vrai, ce ton unique fatiguera un peu, et l'on fera bien de l'interrompre. Du reste, le gris n'est pas heureux pour les instruments d'ivoire comme certaines flûtes, cornets à bouquin et luths, et il assourdit le laiton des cors, trompettes et trombones, ainsi que l'argent des flûtes modernes et le nickel des saxophones. Dans ces cas-là, nous avons remplacé la toile grise par une toile bleu clair, par un velours vert ou même par du bois nu préalablement noirci. Il va sans dire qu'on devra tenir compte de la sensibilité à la lumière.

60 A côté des vitrines verticales, les vitrines-tables ont des avantages bien connus des conservateurs. Elles rapprochent les objets et, pour ainsi dire, permettent d'en faire la lecture ; on peut même s'appuyer légèrement sur le rebord et pencher la tête : n'oublions .pas que l'homme est accoutumé à cette attitude, et il se fatigue vite si on le force a lever 
constamment la tête. Toutefois, le nombre d'instruments qui se prêtent à l'exposition dans les vitrines horizontales est fort restreint. Ce sont les flûtes, pochettes, castagnettes, harmonicas à bouche, guimbardes, en un mot, les instruments petits et peu profonds, qui n'ont pas d'excroissance prononcée, telle que le pavillon des hautbois et des clarinettes et le soufflet de l'accordéon; les vitrines horizontales plus encore que les autres vitrines, demandent des surfaces planes.

61 Avant de clore la série des questions esthétiques, que pose la collection d'exposition, il n'est peut-être pas inutile de constater que tout essai d'emprunter la décoration des salles aux différentes époques et nationalités dont elles renferment les instruments a misérablement échoué. Les parois doivent laisser parler les objets exposés; elles se borneront, pour leur part, à un rôle purement passif. Cependant la paroi la plus sobre doit être peinte, et on évitera l'uniformité ennuyeuse d'une seule couleur pour toutes les salles. Dans ce choix, le conservateur avisé s'inspirera du goût de l'époque, qui, de son côté, a déterminé en son temps l'extérieur de l'instrument et pourra de ce fait lui fournir un cadre approprié. Il se souviendra que la maison bourgeoise des Pays-Bas, à laquelle était destiné le clavecin des Ruckers, avait généralement les parois blanches, que la maison riche de la Renaissance préférait les couleurs plutôt sombres, que le XVIII ${ }^{e}$ aimait les papiers clairs, et il en tirera discrètement des conclusions pour le revêtement coloré des salles correspondantes. Mais qu'on s'abstienne d'ornementation et que l'on proscrive tout ornement «d'époque» et autres enfantillages du genre des concerts de musique ancienne dans le costume du temps !

\section{En marge de l'exposition}

62 À côté de la collection d'exposition, le visiteur trouvera la collection d'études destinée aux recherches approfondies des spécialistes. Ici, au contraire, on donnera la préférence au groupement par espèces, qui permettra une orientation plus rapide et facilitera la comparaison des pièces semblables, corollaire indispensable de toute recherche sérieuse. Dans ce département, les objets pourront être rangés de façon plus compacte et moins artistique. Toutefois, il y aura une différence essentielle entre les salles et les locaux servant de magasins: il faut que les instruments soient entièrement visibles et accessibles, voire même maniables. Les objets ne seront ni parqués ensemble ni entassés les uns sur les autres, et le visiteur n'aura pas à relever les pans de son habit par crainte d'accrocher les objets au passage... En outre, chaque instrument sera muni d'une étiquette. Bref, la collection destinée aux études sera à peu près ce que sont, à l'heure actuelle, les meilleurs des musées d'instruments dans leur collection d'exposition.

Le magasin - ou réserve - ne diffère en aucun point de celui des autres musées. Il requiert une installation qui, au lieu de détruire les objets déposés, les conserve; ces locaux devront donc protéger les instruments contre la poussière, les insectes et les excès et les changements brusques de température. En outre, tout objet portera un numéro en gros chiffres bien lisibles.

Nous en venons maintenant aux questions des étiquettes, du numérotage, des inventaires, des catalogues et des guides. Le problème des inventaires n'offre rien de particulier; on le traitera comme dans les autres musées, et l'on inscrira les acquisitions au moins en double, sur le livre et sur les fiches. Par contre, la question de l'inscription du numéro sur l'objet même demande des précautions spéciales. Il y a trois possibilités : accrocher l'étiquette portant le numéro, la coller sur l'instrument même, ou inscrire le 
numéro directement sur l'objet. Le premier mode, qui a la préférence surtout dans les musées ethnographiques, parce qu'il dispense des longues recherches, souvent si pénibles, quand il s'agit d'objets complexes, a de gros inconvénients dans un musée instrumental, où les objets sont conservés pour être retirés des vitrines et utilisés le plus fréquemment possible. Or, si par ce fait même on risque déjà plus qu'ailleurs de perdre la petite carte portant le numéro, il faut ajouter que, dans la majorité des cas, les seuls endroits permettant le passage du fil, sont précisément ceux dont on a besoin pour le jeu : la lumière des flûtes à bec, les trous latéraux des hautbois, les pistons des cors, etc. Et enfin, le collage des cartes sur l'instrument même, présente deux désavantages. Tout d'abord la carte peut se détacher, et tout conservateur sait ce que cela signifie. Puis, la colle endommage gravement la surface des objets; elle détruit par exemple le vernis si précieux des violons, et l'on n'a pas la possibilité de coller l'étiquette dans un endroit où elle serait invisible. En dépit de tout ce qu'on pourrait trouver à redire, nous nous sommes décidé, dans notre musée, pour la troisième manière: nous avons inscrit les numéros en petits chiffres sur l'objet même, avec de l'encre de noix de galle, qui mord même sur le laiton des trompettes et résiste à l'humidité : le numéro reste invisible pour le commun, il ne peut pas se perdre, et il ne gêne pas le maniement de l'objet. Dans la collection d'étude, cependant, il est préférable, comme nous l'avons indiqué, d'écrire le numéro en chiffres assez gros sur des cartes accrochées, pour faciliter la recherche, soit d'après l'inventaire, soit d'après le catalogue imprimé.

Nous n'insisterons pas sur la question du catalogue. Elle a été traitée maintes fois, et chacun sait combien un catalogue bien fait augmente la valeur d'un musée. Les conservateurs des musées instrumentaux l'ont reconnu de bonne heure. En 1869 déjà, Carl Engel publiait le catalogue des Instruments du South Kensington Museum de Londres, en 1875 Gustave Chouquet faisait imprimer celui du Conservatoire National de Musique à Paris, et en 1888 Victor-Charles Mahillon commençait l'édition de son gros catalogue du Conservatoire Royal de Musique de Bruxelles, qui comprend jusqu'ici cinq volumes (dont les derniers ont été établis en partie par son successeur Ernest Closson), et qui est devenu en quelque sorte la base et le bréviaire de l'organologie de la dernière génération. Plus récemment, dans la génération actuelle, M. Georg Kinsky a fait, en deux gros volumes richement imprimés et illustrés, le catalogue de la collection Heyer (depuis à Leipzig), et l'auteur de la présente étude celui de la Hochschule für Musik de Berlin (1922). Pour continuer la liste, on aurait à citer toutes les collections publiques et particulières nommées au début de cet article : leurs collectionneurs ou conservateurs en ont présenté des catalogues, d'étendue et de valeur inégales il est vrai, mais utiles dans la majorité des cas. Ce serait une tâche des plus séduisantes que de tracer l'histoire du catalogue instrumental, depuis les débuts jusqu'à nos jours, et de suivre l'évolution du criticisme vis-à-vis de l'objet, de la terminologie, de la description, et de la détermination. Et on serait tenté d'y joindre une esquisse des voies nouvelles à frayer dans ce domaine. Sans exposer ici tout ce que devrait contenir un catalogue bien conçu, qu'il nous soit permis de faire appel, sur deux points, au sens critique des auteurs de catalogue. Le conservateur devra être, tout d'abord, le critique de son musée. Il est quelque peu suranné de se faire l'écho de tous les "baptêmes" hasardés des antiquaires. Est-il besoin de citer les exemples trop connus de rédacteurs de catalogues qui vous présentent un violon comme un chef-d'œuvre de Stradivari parce que le marchand y a collé une étiquette de ce nom ? Ou de ceux qui se piquent de posséder un instrument ou même l'instrument sur lequel a joué Mozart, parce que le dit instrument a été acheté à Salzburg ? Et voici l'autre point : il faut, dans un catalogue, qu'on distingue clairement entre les dates et provenances 
marquées, soit au fer rouge, soit sur étiquette, sur l'instrument même, celles qu'on déduit d'un signe quelconque, celles qui sont relevées sur les documents de toute sorte, et enfin celles que nous tirons de la comparaison avec des pièces semblables. La date de 1806 d'un instrument bavarois, par exemple, indiqué comme année fixe, comme terme a quo ou ad quem, peut se déduire : $a$ ) d'une inscription sur l'instrument ; $b$ ) de la couronne au-dessus de la marque, l'électeur de Bavière étant, en 1806, nommé roi ; c) d'une lettre dans laquelle il est fait mention de la pièce; $d$ ) de l'estimation libre du spécialiste. Il est clair que la valeur documentaire de ces quatre données est bien différente; aussi faut-il que, par les signes usuels, comme les guillemets, les parenthèses et les crochets, le cas soit précisé sans équivoque possible. C'est le principe qui est appliqué par exemple dans le catalogue du musée de Berlin.

Le catalogue est, après la " mise en scène ", le second moyen pour faire valoir le musée. Vient ensuite la publication d'un petit guide d'un caractère plus ou moins didactique, depuis le guide tout court qui se borne à diriger le visiteur à travers les salles et à lui indiquer les pièces principales, jusqu'à ceux qui se rapprocheraient d'une sorte de précis d'organologie. Après le guide, il faut mentionner la photo et la carte illustrée vendues à l'entrée ; toute cette publicité rentre dans le cadre de la muséographie générale, et nous pouvons nous dispenser d'en parler. Mais il y a, dans les musées instrumentaux, un facteur de plus : l'audition. Les visites-conférences, qui ont actuellement une si grande importance, demandent une illustration musicale. Elle sera donnée, soit par le conférencier lui-même, soit par un artiste spécialement qualifié, soit par le moyen du gramophone. A côté des visites-conférences, on donne, depuis quelques années, des concerts plus ou moins réguliers, avec les instruments du musée. Et, à l'avenir, on poursuivra dans une voie intermédiaire, réalisée pour la première fois par M. André Schaeffner au département musical du musée ethnographique du Trocadéro à Paris: le concert des disques par haut-parleur.

En terminant cette étude, une dernière question se pose, de nature purement administrative il est vrai, mais bien souvent décisive pour le sort du musée. Rappelons tout d'abord que le premier musée instrumental, conçu pendant la Révolution française, était destiné à faire partie d'un conservatoire national de musique. L'idée a été réalisée quelque soixante-dix ans après, en édifiant le plan de rattachement au Conservatoire de Paris. Une grande partie des collections instrumentales ont été rattachées, comme en France, aux écoles musicales : ainsi les musées de Berlin, de Bruxelles, de Florence, de Milan et de Prague ; à Londres, la Collection Donaldson, tout au moins, a été donnée à la Royal Academy of Music. Les collections américaines sont reliées aux universités, il en est de même pour le musée de Leipzig. Mais ce dernier cas est un peu différent : à Leipzig, il s'agit d'une liaison avec la musicologie pure, tandis qu'en Amérique, les cours universitaires se rapportent plutôt aux éléments de la musique pratique - c'est une sorte de conservatoire, dans le cadre de l'université, pour les étudiants de toutes les facultés. La collection d'Eisenach fait partie du "Bachhaus", domicile de Jean-Sébastien Bach; Markneukirchen et Stockholm ont des musées indépendants. Toutes les autres collections instrumentales sont incorporées dans des musées généraux. On pourra se demander laquelle de ces solutions est la meilleure?

Chacune, à vrai dire, a ses avantages particuliers. Les conservatoires permettent au musée un contact vivant avec les étudiants de la musique qui, adroitement guidés, pourront en profiter pour élargir leur horizon, pour enrichir leur éducation, hélas souvent négligée, et pour former leur style. Les universités, à leur tour, offrent, par le moyen d'un musée 
instrumental, un matériel précieux, indispensable aux études musicologiques et qui permet d'incorporer la musicologie dans les différentes branches de l'histoire de l'humanité. Les musées généraux, enfin, amènent un public, de hasard d'abord, et intéressé ensuite, qui resterait étranger aux collections instrumentales si celles-ci demeuraient a part.

Par ailleurs toutes ces solutions souffrent d'un même inconvénient: c'est le rôle de Cendrillon qu'on leur fait généralement jouer sous une direction imposée et qui n'est pas toujours préparée ou même favorable à la musicologie. Le directeur du conservatoire est presque toujours compositeur ou virtuose et par là, bien souvent peu soucieux du développement d'une institution dont il ne sait pas se servir, et qui n'usurpe, à son avis, que trop d'espace, utilisable d'une manière plus conforme aux buts d'une école. Il n'a, de plus, guère de compétence en ce qui concerne les conditions et nécessités d'un musée. Le directeur d'un musée d'art ou d'histoire connaîtra ces exigences ; mais, personnellement, il aura son centre d'intérêt dans l'histoire de l'art et, même si nous lui supposons une certaine bienveillance à l'égard du département musical confié à ses soins, il subira inévitablement l'influence de l'intérêt prédominant que manifeste la majorité du public pour les tableaux et les sculptures, en regard des instruments de musique. Nous retombons ici dans l'un de ces éternels " problèmes des minorités ». Ce problème fâcheux subsiste même dans le cas où le musée instrumental est indépendant, localement et administrativement. Ici la collection joue un peu le rôle de Cendrillon auprès d'un directeur intermédiaire ou auprès du ministre compétent : l'intérêt administratif est pesé à la balance de l'intérêt public, dont les poids sont représentés par les chiffres d'entrées. En d'autres termes : toutes les économies à faire, et aujourd'hui il ne s'agit guère que de cela, frapperont en premier lieu les " minorités », quelle que soit leur valeur.

Comme en tout autre domaine, la seule puissance capable de réduire à l'absurde la statistique, c'est la personnalité qui fraye le chemin à sa cause. Aucune des solutions administratives n'est bonne par elle-même, sans celui qui en sait tirer tous les avantages et en éviter tous les inconvénients; aucune n'est assez mauvaise pour immobiliser ou entraver un véritable chef. Et pour conclure, il faut reconnaître que c'est l'homme qui décide, et que tous les principes qu'on peut donner sont plutôt des expériences et des méthodes personnelles que des recettes à suivre invariablement. L'avenir des musées instrumentaux ne dépend ni du budget ni de l'organisation administrative, mais de la qualité de leurs conservateurs, de leur énergie, de leurs connaissances, de leur horizon et de leur goût.

\section{BIBLIOGRAPHIE}

BERNER Alfred, 1984, « Die alte Musikinstrumenten-Sammlung in Berlin », Wege zur Musik, Staatliches Institut für Musikforschung Preussischer Kulturbesitz, Berlin, ,oir en particulier pp. 75-111.

KARP Cary (ed.), 1992, The Conservation and Technology of Musical Instruments. A Bibliographic Supplement to Art and Archeology Technical Abstracts, Volume 28. 
BARCLAY Robert L. (ed.), 1997, The Care of Historic Musical Instruments. CCI, Museums \& Galleries Commission, CIMCIM.

\section{Bibliographie sélective de Curt Sachs (Berlin 1881 - New York 1959) 6}

HORNBOSTEL Erich Maria von und Curt SACHS, 1914, « Systematik der Musikinstrumente: ein Versuch ». Zeitschrift für Ethnologie (Berlin) XLVI, 4-5: 553-590. [Trad. anglaise (1961): Classification of Musical Instruments. Translated from the original German by Anthony Baines and Klaus Wachsmann. The Galpin Society Journal XIV: 3-29. Rééd. (1992) in Helen Myers, ed.: Ethnomusicology: An Introduction: 444-461. Adaptation française (1978) : Les instruments de musique du monde entier. Une encyclopédie illustrée conçue par Diagram Group. Paris: Albin Michel.]

SACHS Curt, 1910, Musik und Oper am kurbrandenburgischen Hofe. Berlin: J. Bard. [Rééd. (1977): Hildesheim/New York: Georg Olms Verlag.]

SACHS Curt, 1913, Reallexikon der Musikinstrumente. Berlin: Felber. [Rééd. (1972/1979): Hildesheim, New York: Georg Olms Verlag.]

SACHS Curt, 1915, Die Musikinstrumente Indiens und Indonesiens, zugleich eine Einführung in die Instrumentenkunde. Berlin: Georg Reimer. Handbücher der Königlichen Museen zu Berlin. [Rééd. (1983): Hildesheim, New York: Georg Olms Verlag.]

SACHS Curt, 1921, Die Musikinstrumente des alten Ägyptens. Berlin: Staatliche Museen zu Berlin, Mitteilungen aus der ägyptischen Sammlung, 3.

SACHS Curt, 1922, Sammlung alter Musikinstrumente bei der Staatlichen Hochschule für Musik zu Berlin. Beschreibender Katalog. Berlin.

SACHS Curt, 1923, Die modernen Musikinstrumente. Berlin: Max Hesses Handbücher, Bd. 68.

SACHS Curt, 1929, Geist und Werden der Musikinstrumente. Berlin. [Rééd. (1965): Hilversum, F.A.M. Knuf; rééd. (1975): Buren: Fritz Knuf.]

SACHS Curt, 1930a, Handbuch der Musikinstrumentenkunde. Leipzig: VEB Breitkopf \& Härtel Musikverlag (Kleine Handbücher der Musikgeschichte nach Gattungen, Bd. 12). [Rééd. (1967): Hildesheim: Georg Olms; rééd. (1979): Leipzig, Breitkopf \& Härtel.]

SACHS Curt, 1930b, Vergleichende Musikwissenschaft in ihren Grundzügen. Leipzig.

SACHS Curt, 1933, Eine Weltgeschichte des Tanzes. Berlin: Georg Reimer [Rééd. (1976/1984): Hildes heim, Zürich, New York: Georg Olms Verlag. Trad. anglaise (1976): World History of the Dance. Tr. By Bessie Schönberg. Hildesheim, New York : Georg Olms Verlag. Trad. française (1938) : Histoire de la danse. Trad. par L. Kerr. Paris : Gallimard, avec seulement la moitié des illustrations et une bibliographie sélective.]

SACHS Curt, 1934, «La signification, la tâche et la technique muséographique des collections d'instruments de musiques ». Mouseion 27/28:5-36.

SACHS Curt, 1938, Les instruments de musique de Madagascar. Paris : Institut d'Ethnologie. Travaux et mémoires de l'Institut d'Ethnologie XXVIII.

SACHS Curt, 1939, Vergleichende Musikwissenschaft - Musik der Fremdkulturen. 2. Neubearb, Aufl. Heidelberg: Quelle und Meyer, 1959 (Musikpädagogische Bibliothek 2.).

SACHS Curt, 1940, The History of Musical Instruments. London: Dent. [Rééd. (1968): New York: W.W. Norton]. 
SACHS Curt, 1943, The Rise of Music in the Ancient World, East and West. New York: W.W. Norton.

SACHS Curt, 1953, Rhythm and Tempo: A Study in Music History. New York: W.W. Norton.

SACHS Curt, 1955, Our Musical Heritage. A Short History of Music. New York: Prentice Hall.

SACHS Curt, 1956, A Short History of World Music. London: Dobson.

SACHS Curt, 1962 , The Wellsprings of Music. An Introduction to Ethnomusicology. The Hague: Martin Nijhoff.

SACHS Curt, 1968, Die Musik der Alten Welt in Ost und West. Berlin : Akademie-Verlag.

SACHS Curt et al., 1977, The Place of Musicology in American Institutions of Higher Learning. New York : Da Capo Press.

\section{NOTES}

1. Article précédemment publié dans la revue Mouseion, vol. 27-28, 1934, pp. 153-184, réédité avec l'aimable autorisation de Madame Gabrielle Forrest, fille de Curt Sachs.

2. Voir Berner 1984: 75-111 (chapitre «Wissenschaft und Forschung. Curt Sachs 1919-1933»).

3. Voir notamment les travaux du CIMCIM (Comité international des musées et collections d'instruments de musique) et tout particulièrement Karp 1992 et Barclay 1997.

4. Les intertitres sont de la rédaction.

5. Geist und Werden des Musikinstrumente, Berlin 1929

6. Bibliographie établie par Laurent Aubert.

\section{RÉSUMÉS}

Dans un style à la fois clair, savant et d'une grande qualité littéraire, Curt Sachs nous livre ici un véritable précis de muséographie à destination des organologues et des responsables de collections d'instruments de musique. Publiée pour la première fois en 1934, cette contribution trouve encore aujourd'hui toute sa place dans un dossier consacré à la musique dans les musées. En effet, les réflexions qu'il nous soumet sur la conservation et la restauration des instruments de musique, sur leur exposition et sur des questions touchant à l'iconographie et à la scénographie, conservent une grande partie de leur actualité. Peu d'organologues après lui ont en outre traité ce sujet avec une telle maîtrise et un tel souci du détail. Pour peu qu'on les replace dans leur contexte, les recommandations de l'ancien Directeur du Musée instrumental de Berlin - destitué de sa position par le régime nazi une année avant la publication de cet article méritent qu'on les considère avec la plus grande attention (L.A.). 


\section{AUTEUR}

\section{CURT SACHS}

Curt Sachs (Berlin, 1881 - New York, 1959) fit ses études à l'Université de Berlin et se consacra à la musicologie à partir de 1909. Nommé directeur de la Collection nationale d'instruments (Staatliche Instrumenten-Sammlung) à Berlin en 1919, il enseigna également à l'Université, à la Hochschule für Musik, à l'Akademie für Kirchen- und Schulmusik. En 1933 il quitta l'Allemagne. Jusqu'en 1937 il fut attaché au Musée de l'Homme à Paris et fit des conférences à la Sorbonne, puis il s'installa à New York et enseigna dans diverses grandes universités américaines, à Columbia University en dernier lieu. Excellent professeur, musicologue aux idées particulièrement originales et auteur particulièrement prolixe, Sachs a eu sur la musique des vues d'ensemble fécondes. Unissant l'un des premiers ses aspects morphologiques, ethnologiques et historiques, il a jeté les bases de nombreuses recherches nouvellesfr 